\title{
Quality assurance of simultaneous treatment of two targets in pelvic region planned with single isocenter using three dimensional conformal radiotherapy (3DCRT) technique
}

\author{
Suman Kumar Putha ${ }^{1}$, Challapalli Srinivas' ${ }^{1}$ Vadhiraja Bejadi Manjunath ${ }^{2}$, Arun Elavunkal Sukumaran ${ }^{1}$, \\ Sridhar Chinthamani ${ }^{3}$, Prakash Saxena ${ }^{1}$, Sourjya Banerjee ${ }^{1}$, Dinesh Pai Kasturi ${ }^{1}$ \\ ${ }^{1}$ Department of Radiotherapy \& Oncology, Kasturba Medical College Hospital, Mangalore, India \\ ${ }^{2}$ Department Radiation Oncology, Manipal Hospital, Bangalore, India \\ ${ }^{3}$ Department of Radiation Oncology, Father Muller Oncology Center, Mangalore, India
}

Received April 07, 2015; Revised May 27, 2015; Accepted May 29, 2015; Published Online July 17, 2015

\section{Technical Report}

\begin{abstract}
Purpose: The purpose of this study was to conduct quality assurance of a three dimensional conformal radiotherapy (3DCRT) of two targets in pelvis region planned with single isocenter technique. Methods: A treatment plan was generated with two identical water phantoms with ionization chamber (IC) sleeves (IC-1 \& IC-2), simulated as if targets are in pelvis region, simultaneously irradiated with single isocenter technique with a dose prescription of $300 \mathrm{cGy}$ for point dose verification. A two dimensional ion chamber array detector was used for fluence verification. Results: Calculated minimum, mean and maximum dose (in cGy) for IC-1 \& IC-2 were 295, 303 and 307 as per dose volume histogram. The global dose maximum was found to be 307.4 cGy. Measured point doses to both lesions were within $\pm 2.5 \%$ of the computed dose. A pass percentage of $97 \%$ was obtained with the set of criteria $3 \mathrm{~mm}$ distance to agreement and $3 \%$ dose difference for fluence verification. Conclusion: Treatment execution of two targets simultaneously with single isocenter can reduce positional errors and delivery time.
\end{abstract}

Keywords: Quality Assurance; Two Targets; Single Isocenter; Conformal Radiotherapy

\section{Introduction}

Solid tumours in pelvic region present with bilateral hip bone metastases where critical organs (e.g. bladder and rectum) need to be spared while necessitating their simultaneous radio therapeutic treatment. Such treatments are usually executed using modern radiotherapy techniques such as intensity modulated radiotherapy (IMRT), stereotactic body radiation therapy (SBRT), rapid arc, volumetric arc radiotherapy (VMAT), cyberknife ${ }^{\circledR}$ and tomotherapy. In convention, multiple targets can be treated individually with a set of beams having their own isocenter. Treatment planning and execution of multiple targets results in prolongation of treatment time [starting from pre-treatment quality assurance (QA), patient positioning, and setup corrections for every target treatment and treatment delivery]. Another method is to have a common isocenter, around which the gantry rotates and delivers the radiation to multiple target sites, one at a time. This method can considerably reduce the time of pre-treatment plan QA and execution. Many investigators have verified the dosimetric quality of a common isocentric plan to treat multiple tumours especially in brain metastatic lesions.
Potter et al. ${ }^{1}$ have investigated the treatment of multiple brain tumours with 4 bank micro multi leaf collimator (mMLC) and have found that the single isocentric treatment plan was as good as multiple isocentric plan, which can reduce considerable amount of time in QA \& treatment delivery. Luxton et al. ${ }^{2}$ treated non-spherical targets in brain with single isocenter and achieved good dose conformity. Clark et al. ${ }^{3}$ have verified the feasibility of single isocenter in VMAT radiosurgery, for the treatment of multiple brain metastasis and concluded that it is a better method. Huang et al. ${ }^{4}$ have compared the quality of target coverage and dose conformity with single isocentric VMAT-SRS plans to dynamic conformal arc therapy (DCAT) and they concluded that the DCAT technique may result in larger low dose regions.

Many authors have studied the validity of single isocentric plans and concluded that it could be a better option in saving pre-treatment QA time as well as treatment delivery time with less systematic errors..$^{5-7}$ Marks et al. have confirmed through their investigation that three dimensional conformal radiotherapy (3DCRT) technique can be a possible alternative

Corresponding author: Challapalli Srinivas; Department of Radiotherapy \& Oncology, Kasturba Medical College Hospital, Mangalore, India.

Cite this article as: Putha SK, Srinivas C, Vadhiraja BM, Sukumaran AE, Chinthamani S, Saxena P, Banerjee S, Pai-Kasturi D. Quality assurance of simultaneous treatment of two targets in pelvic region planned with single isocenter using three dimensional conformal radiotherapy (3DCRT) technique. Int $J$ Cancer Ther Oncol 2015; 3(3):3315. DOI: 10.14319/ijcto.33.15

[A part of this research was presented at $13^{\text {th }}$ Asia-Oceania Congress of Medical Physics (AOCMP) \& $11^{\text {th }}$ South - East Asian Congress of Medical Physics (SEACOMP), which was held from December 12-14, 2013 in Singapore] 
to radiosurgery with fixed shaped coplanar or non-coplanar techniques with wedged radiation fields having beams conformed to irregular shaped intracranial lesions, as the goal of both the techniques is to achieve better dose conformity. ${ }^{8}$ Similar logic can be used to treat multiple lesions simultaneously with different/single beam sets conformed to different lesion sites elsewhere extra cranially which can save pre-treatment QA time \& treatment time appreciably. Planning of these techniques with 3DCRT using treatment planning system (TPS), requires a logical approach (different beam sets conformed to multiple lesions sharing a common isocenter, having different weight points, the feasibility/flexibility to use different wedge angles using motorized wedge option, to obtain better conformal dose coverage. ${ }^{9-11}$ The objective of this study is to validate a single isocentric plan using 3DCRT treatment planning technique in terms of dose conformity and coverage for the treatment of two pelvic metastatic lesions (simulated in water phantom) using composite point dose method and two dimensional (2D) ion chamber array detector.

\section{Methods and Materials}

A case of carcinoma of lung with bilateral hip bone metastases (targets) in pelvic region was planned for radiotherapy using common isocenter using 3DCRT technique with CMS $\mathrm{XiO}$ TPS with superposition algorithm (Figure 1). Treatment was executed with medical linear accelerator (Elekta Compact; Stockholm, Sweden), using $6 \mathrm{MV}$ photon beam at a dose rate of $350 \mathrm{MU} / \mathrm{min}$ with 40 pair multi leaf collimator (MLC) leaves (projected leaf width $1.0 \mathrm{~cm}$ at isocenter) arranged in two banks and also having motorized wedge facility.

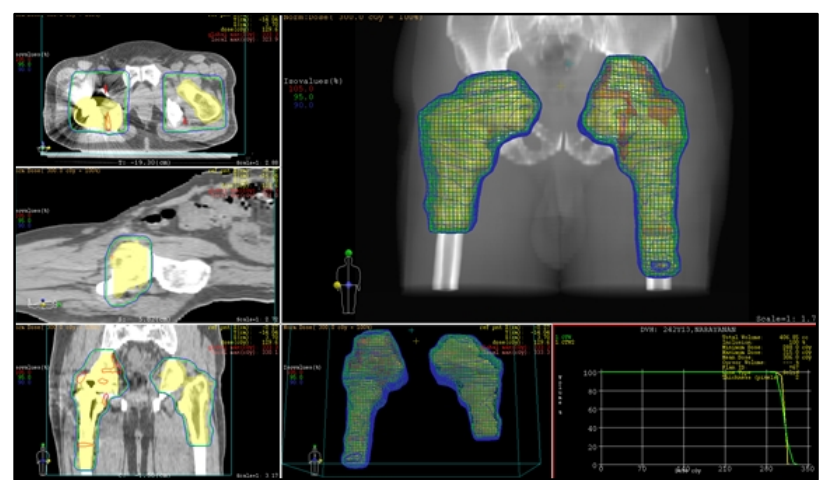

FIG. 1: Treatment planning with common isocenter using 3DCRT technique.

\section{Quality assurance (QA)}

\section{Point dose verification}

For the point dose verification (composite dosimetry) of this plan, same patient geometry was simulated by two identical water phantoms (having dimensions of $30 \mathrm{~cm} \times 15 \mathrm{~cm} \times 15$ $\mathrm{cm}$ each) with $0.65 \mathrm{cc}$ ionization chamber (Scanditronix
Farmer Type Chamber FC65) (IC) sleeves were placed side by side, having surface fiducials. The active volumes of the two ionization chambers were used to simulate the targets. A planning computed tomography (CT) scan was acquired along with ICs placed inside the sleeves (Figure 2) and the images were exported to the contouring station (CMS Focal Sim).

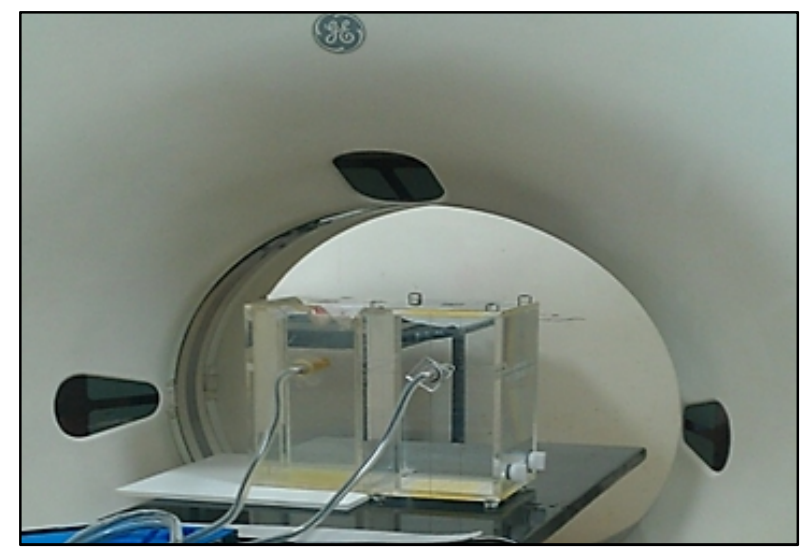

FIG. 2: CT Simulation of identical water phantoms with ionization chambers (IC-1 \& IC-2) kept inside the sleeves.

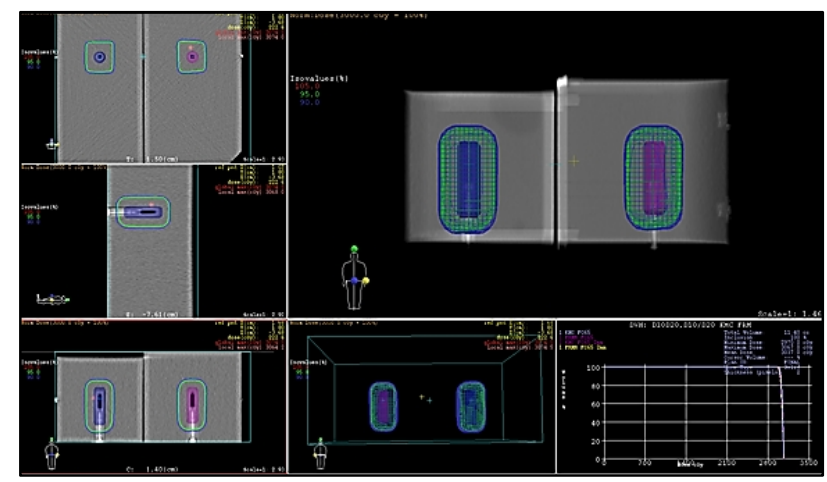

FIG. 3: Treatment planning of IC-1 \& IC-2 with single isocenter.

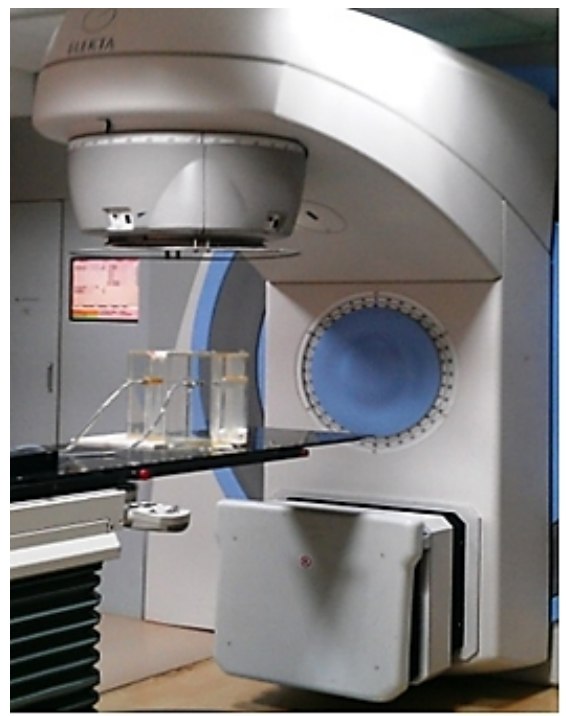

FIG. 4: Point dose verification with water phantom and ICs. 
The chamber positions were contoured as IC-1 \& IC-2 simulating the bilateral targets in the pelvis in an actual patient. A combined target (IC-3) was generated with a $5 \mathrm{~mm}$ margin encompassing both IC-1 \& IC-2. Contoured CT data set was exported to CMS XiO TPS for beam placement and dose calculations. A group of six main beams were placed with centre of IC-3 as isocenter such that three beams with gantry angles $180^{\circ}, 270^{\circ}$ and $0^{\circ}$ conformed to IC- 1 \& three beams with gantry angles $0^{\circ}, 90^{\circ}$ and $180^{\circ}$ conformed to IC-2. The field in field (sub beam) technique was used with different MLC leaf positions to eliminate the hot spots. In order to obtain uniform dose distribution around the targets, appropriate beam weights, weight points (placed inside the targets) and different wedge angles were chosen. Superposition algorithm was used for dose calculations. A dose of 300 cGy is prescribed to the $100 \%$ isodose line that is covering both the targets. The calculated monitoring units (MU) for each field was normalized to the specified weight points and were adjusted such that each target was covered with $95 \%$ of isodose line. The generated plan (Figure 3) was exported and executed (Figure 4) using Mosaiq ${ }^{\circledR}$ record and verification system for point dose verification.

The measurement was carried out with water phantoms and ICs placed inside the sleeves which were connected to the electrometers (Scanditronix DOSE1). The measured charge collected from each electrometer was converted to absorbed dose after applying correction factors of temperature \& pressure (КтР), polarization $\left(\mathrm{K}_{\mathrm{pol}}\right)$, saturation (Ksat), beam quality (KQQo) and calibration factor( $\mathrm{NDW}_{\mathrm{DW}}$ of the chamber.

\section{Two dimensional (2D) verification}

A 2D ion chamber array detector (I'matriXX) was used for planar dose verification. This device consists of a 1020 vented ion chamber array detectors arranged in $32 \times 32$ grids. The each chamber volume is $0.08 \mathrm{~cm}^{3}$ with the height of $5 \mathrm{~mm}$ and diameter of $4.5 \mathrm{~mm}$. The maximum dose rate detectable by the detectors is $5 \mathrm{~Gy} / \mathrm{min}$ and minimum of $0.1 \mathrm{~Gy} / \mathrm{min}$. The bias voltage required for the matrix system is $500 \pm 30 \mathrm{~V}$. The maximum field of view is $24 \times 24 \mathrm{~cm}^{2}$. The matrix device can be directly connected to computer via standard Ethernet interface to acquire the measurements. The I'matriXX device with $5 \mathrm{~cm}$ solid water phantom (SP-34) positioned above it was scanned with $2 \mathrm{~mm}$ CT slice thickness. In order to verify the TPS generated plan, a verification plan was produced with CT data of the detector system to estimate the fluence. In the verification plan, all gantry and collimator angels were set to 0 degrees and exported to the scanned detector system with the detector plane positioned at isocenter. Generated verification plan was exported and executed (Figure 5) using Mosaiq ${ }^{\circledR}$ record and verification system for planar dose verification with I'matriXX device.

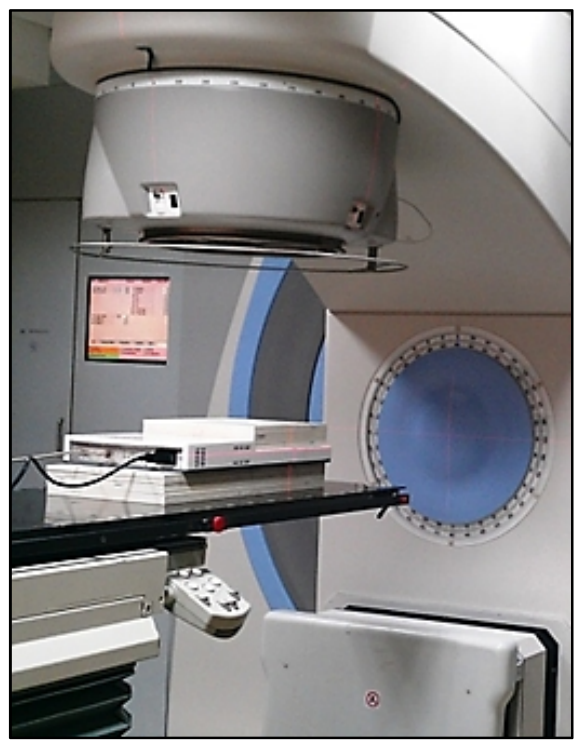

FIG. 5: 2D dose verification with I'MatriXX.

The beam central axis was made perpendicular to the I'matriXX measurement level at the center of the measurement area during the measurement. By executing the verification plan, the cumulative fluence at the detector plane was calculated and transferred to the Omnipro software for comparison. Dose distributions obtained with I'matriXX was rescaled at $0.1 \mathrm{~cm}$ resolution using OmniPro IMRT analyzing software. All measured fluence was compared with TPS dose plane by $2 \mathrm{D}$ gamma evaluation using $3 \%$ dose difference and $3 \mathrm{~mm}$ distance-to-agreement (DTA) criteria.

\section{Results}

The mean meter readings $(\mathrm{M})$ obtained by point dosimetry method with two 0.6 cc FC65 ionization chambers (IC-1 \& IC-2) are shown in Table 1.

The final dose measurements were obtained using NDw based formalism ${ }^{12}$ and compared with the TPS calculated values as shown in Table 2.

A deviation of $-1.87 \%$ and $-1.30 \%$ for IC-1 and IC-2 target was observed from the calculated value. The minimum, maximum and mean dose (cGy) covered by the IC-1 \& IC-2 volumes were 295, 307 and 303 as per dose volume histogram index from the generated treatment plan. The global dose maximum obtained from the plan was 307.4 cGy. Measured point doses to both lesions were within $\pm 2 \%$ of the TPS calculated dose. A pass percentage of $97 \%$ was obtained with the set criteria of $3 \mathrm{~mm}$ distance to agreement (DTA) and 3\% dose difference for fluence verification (Figure 6). 
TABLE 1: Cumulative charge collected by IC's.

\begin{tabular}{|c|c|c|c|c|c|c|}
\hline \multirow{3}{*}{$\begin{array}{l}\text { Gantry } \\
\text { angle }\end{array}$} & \multirow[t]{3}{*}{ Beam description } & \multirow{3}{*}{$\begin{array}{c}\text { MU's } \\
\text { delivered }\end{array}$} & \multicolumn{4}{|c|}{ Cumulative charge $\left({ }^{*} \mathrm{nC}\right)$ collected } \\
\hline & & & \multicolumn{2}{|c|}{ IC-1 } & \multicolumn{2}{|c|}{ IC-2 } \\
\hline & & & Trial-1 & Trial-2 & Trial-1 & Trial-2 \\
\hline $270^{0}$ & RT LAT DIR WEDGE & 153 & 6.93 & 6.92 & 18.36 & 18.20 \\
\hline $0^{0}$ & RT ANT DIR WEDGE & 196 & 7.02 & 7.01 & 36.76 & 36.55 \\
\hline $0^{0}$ & RT ANT DIR SUB & 25 & 7.04 & 7.02 & 40.67 & 40.38 \\
\hline $0^{0}$ & LT ANT DIR WEDGE & 232 & 10.18 & 10.16 & 40.69 & 40.36 \\
\hline $0^{0}$ & LT ANT DIR SUB1 & 20 & 14.74 & 14.71 & 40.70 & 40.34 \\
\hline $0^{0}$ & LT ANT DIR SUB2 & 29 & 30.48 & 30.43 & 40.80 & 40.31 \\
\hline $90^{0}$ & LT LAT DIR WEDGE & 203 & 46.47 & 46.27 & 46.98 & 46.27 \\
\hline $180^{\circ}$ & LT POST DIR SUB & 41 & 46.56 & 46.36 & 57.34 & 46.27 \\
\hline $180^{\circ}$ & LT POST DIR WEDGE & 415 & 46.59 & 46.39 & 61.10 & 60.24 \\
\hline $180^{\circ}$ & RT POST DIR SUB & 20 & 59.91 & 59.67 & 61.24 & 60.24 \\
\hline $180^{\circ}$ & RT POST DIR & 109 & 61.87 & 61.62 & 61.25 & 60.24 \\
\hline \multicolumn{3}{|c|}{ Mean Meter Reading (M) } & \multicolumn{2}{|c|}{61.75} & \multicolumn{2}{|c|}{60.75} \\
\hline
\end{tabular}

${ }^{*} \mathrm{nC}=$ nano Coulombs

TABLE 2: Percentage deviation between measured and TPS calculated point dose.

\begin{tabular}{|c|c|c|c|c|c|}
\hline \multicolumn{2}{|c|}{ Measured Mean dose (cGy)= M x TCF@ } & \multicolumn{2}{|c|}{ TPS calculated Mean dose (cGy) } & \multicolumn{2}{|c|}{ Percentage Deviation (\%) } \\
\hline IC-1 & IC-2 & IC-1 & IC-2 & IC-1 & IC-2 \\
\hline 297.4 & 299.1 & 302.8 & 303.0 & -1.78 & -1.29 \\
\hline
\end{tabular}

$@ \mathrm{TCF}=$ Total Correction Factor (NDwx KTP x KpolX KsatX KQQo ${ }^{12}$

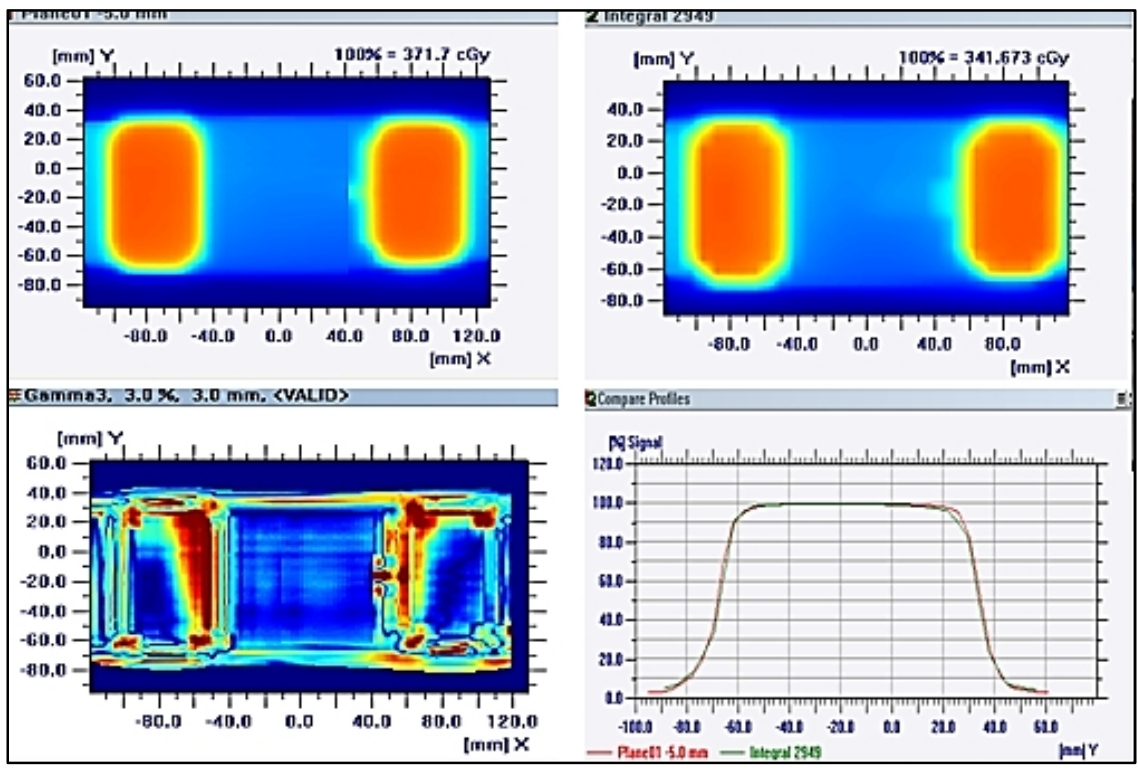

FIG. 6: Evaluation of 2D fluence verification using I'mRTMatriXX ${ }^{\mathrm{TM}}$ device.

\section{Discussion}

In the present study, treatment planning of two targets in pelvic region simultaneously treated with common isocenter using 3D-CRT technique was studied and the delivery accuracy was checked in terms of point dose and fluence measurements. Earlier a similar kind of attempt has been made by several investigators for the treatment of multiple intracranial lesions using highly sophisticated state of art of radiotherapy with algorithms based on inverse planning. ${ }^{1-7} \mathrm{An}-$ other group of investigators have tried to achieve the same target using 3DCRT techniques to treat multiple intracranial lesions with coplanar \& non-coplanar beams. ${ }^{8}$ Keeping the ultimate goal of radiotherapeutic treatment in view, i.e., to achieve maximum target/CTV dose coverage (95\% -107\% isodose coverage to CTV/PTV) \& conformity with maximum possibility of normal tissue sparing, the idea of treating two/multiple targets (intra or extracranial) simultaneously having a common isocenter is always appreciable in terms of time consumed in pre-treatment QA and treatment delivery as well to minimize the setup errors. In contrast the treatment plans with multiple isocenter are time consuming, error prone, and may attribute many uncertainties in the setup and positioning resulting in large systematic errors in the treatment delivery. 
Our study was based on the dose computed in the XiO TPS, which employs superposition algorithm. Researchers ${ }^{13-16}$ have shown that computed dosimetric results from one dose calculation algorithm can vary from that of other dose calculations algorithms such as Acuros $\mathrm{XB}^{13,14}$, analytical anisotropic algorithm ${ }^{14,15}$, and collapsed cone convolution superposition algorithm ${ }^{16}$. The validation of a single isocentric plan for two targets needs to be further investigated in other TPSs with different dose calculation algorithms.

\section{Conclusion}

As the composite point dosimetry results were in agreement with the TPS calculated dose, the method adopted for treating two targets with common isocenter simultaneously with 3D-CRT technique is a better option to reduce the pre-treatment QA and treatment time significantly, at the same time achieving the required coverage as in other sophisticated techniques and higher state of art equipments in the field of Radiotherapy. This technique can be further applied to treat multiple targets simultaneously with different fraction sizes \& doses. This can significantly help in radiobiological control of gross and distant lesions (if any).

\section{Conflict of interest}

The author declares that they have no conflicts of interest. The authors alone are responsible for the content and writing of the paper.

\section{References}

1. Potter L, Lian J, Morris D, et al. Treating multiple tumors simultaneously with a 4-bank mMLC. Available from http://www.med.unc.edu/radonc/proj/treating-mul tiple-tumors-simultaneously-with-a-4-bank-mmlc

2. Luxon G, Jozef G. Luxton G, Jozsef G. Single isocenter treatment planning for homogeneous dose delivery to nonspherical targets in multiarc linear accelerator radiosurgery. Int J Radiat Oncol Biol Phys. 1995;31:635-43.

3. Clark GM, Popple RA, Young PE, Fiveash JB. Feasibility of single iso-center volumetric modulated arc radiosurgery for treatment of multiple brain metastases. Int J Radiat Oncol Biol Phys. 2010; 73:296-302.

4. Huang C. Treatment of multiple brain metastases using stereotactic radiosurgery with single isocenter volumetric arc therapy: Comparison with conventional dynamic conformal arc and static beam stereotactic radiosurgery; Master's thesis submitted to Duke University 2012. Available from http://dukespace.lib.duke.edu/dspace/bitstream/han dle/10161/5478/Huang_duke_0066N_11362.pdf?se quence $=1$

5. Ebert MA, Zavgorodni SF, Kendric LA, et al. Multi-isocenter stereotactic radiotherapy: implications for target dose distributions of systematic and random localization errors. Int J Radiat Oncol Biol Phys. 2001;51:545-54.

6. Shrtaus N, Schifter D, Alani S, et al. Stereotactic Treatment of multiple Targets using single Isocenter: Planning, Dosimetric and Deliver advantages. Med Phys. 2011;38: 3395.

7. VanderSpek L, Wang J, Alksne J, Murphy KT. Single fraction, single isocenter intensity modulated radiosurgery (IMRS) for multiple brain metastases: Dosimetric and early clinical experience. Int J Radiat Oncol Biol Phys. 2007; 69:265.

8. Marks LB, Sherouse GW, Das S, et al. Conformal radiation therapy with fixed shaped coplanar or non-coplanar radiation beam bouquets: a possible alternative to radiosurgery. Int J Radiat Oncol Biol Phys. 1995;33:1209-19.

9. Ramani R, O'Brien PF, Davey P, et al. Implementation of multiple isocenter treatment for dynamic radiosurgery. Br J Radiol. 1995; 68:731-5.

10. Sherouse GW. A mathematical basis for selection of wedge angle and orientation. Med Phys. 1993; 20:1211-8.

11. Sherouse GW, Bourland JD, Reynolds K, et al. Virtual Simulation in the clinical setting: some practical considerations. Int J Radiat Oncol Biol Phys. 1990; 20:1059-65.

12. An international code of practice for dosimetry based on absorbed dose to water IAEA Tech. Series No.398, Absorbed dose determination in external beam radiotherapy. International atomic energy agency, Vienna: IAEA; 2000.

13. Ojala J. The accuracy of the Acuros XB algorithm in external beam radiotherapy - a comprehensive review. Int J Cancer Ther Oncol. 2014; 2:020417.

14. Rana S, Rogers K, Pokharel S, Cheng C. Evaluation of Acuros XB algorithm based on RTOG 0813 dosimetric criteria for SBRT lung treatment with RapidArc. J Appl Clin Med Phys. 2014; 15:4474.

15. Rana S. Clinical dosimetric impact of Acuros XB and analytical anisotropic algorithm (AAA) on real lung cancer treatment plans: review. Int J Cancer Ther Oncol. 2014; 2:02019.

16. Oyewale S. Dose prediction accuracy of collapsed cone convolution superposition algorithm in a multi-layer inhomogenous phantom. Int J Cancer Ther Oncol. 2013; 1:01016. 